\title{
Transstadial transmission and maintenance of Francisella tularensis in the Blacklegged Tick Ixodes scapularis.
}

\author{
Rinosh J Mani ${ }^{1,2^{*}}$, Kenneth D Clinkenbeard ${ }^{1}$ \\ ${ }^{1}$ Department of Veterinary Pathobiology, Center for Veterinary Health Sciences, Oklahoma State University, Stillwater, OK 74078 \\ United States of America \\ ${ }^{2}$ Veterinary Diagnostic Laboratory, Michigan State University, Lansing, MI 48910, United States of America
}

\begin{abstract}
In the south-central United States tularemia endemic region, approximately $10 \%$ of human cases occur during the fall-winter season when the questing activity of its primary tick vectors for this region Dermacentor variabilis and Amblyomma americanum is minimal. It is also been reported that approximately $40 \%$ of tularemia infected domestic cats contract the disease during the fall-winter season. To more fully characterize the potential role of $I$. scapularis, whose host seeking activity peaks during the fall-winter season in the south-central United States, as a vector for tularemia, the biology of $F$. tularensis subspecies holarctica strain LVS in colonyreared $I$. scapularis artificially infected with LVS by capillary feeding was studied. Capillary-fed I. scapularis larva and nymph were initially colonized with $1.9 \times 10^{3}$ and $1.1 \times 10^{4} \mathrm{CFU} /$ tick, respectively. For nymphs molted from colonized larvae, the percent of molted nymphs colonized declined at the time of molting, but LVS persisted in $15 \%$ of molted nymphs up to 175 days post-capillary feeding. In contrast, only $5 \%$ of adults molted from infected nymphs maintained LVS colonization through 150 days post-capillary feeding. For capillary-fed adults, LVS initially colonized the gut and disseminated to hemolymph and salivary glands by 14 day post-feeding. Post-capillary feeding larvae and nymphs maintained LVS colonization initially and persisted although in a low percentage in molted nymphs and molted adults. Based on the feeding habits of $I$. scapularis adults, this tick species has the potential to act as a bridging vector for human tularemia for fall-winter cases of tularemia.
\end{abstract}

Keywords: Ixodes scapularis, Francisella tularensis, Tularemia, Transstadial transmission.

Accepted August 16, 2018

\section{Introduction}

Francisella tularensis is a highly infectious gram-negative coccobacillus which causes the disease tularemia in humans and a wide range of vertebrate and invertebrate animals [1]. There are two main subspecies of this bacterium, namely subspecies tularensis exclusively seen in the North America and subspecies holarctica seen in North America as well as in Eurasia [2,3]. Molecular subtyping have identified a number of genotypes for the two subspecies of which genotype A1b seems to be the most virulent with a case fatality rate of $25 \%$ and is associated with tick borne infections [4-6]. Historically, human tularemia cases were associated with handling of dead/sick rabbits; however, in recent years it is manifested as a tick borne disease where the ulceroglandular form of the disease predominates [2,7-9].

In the south-central tularemia endemic region of United States, the tularemia agent Francisella tularensis is maintained via a spring-summer tick-small mammalian enzootic cycle coincident with questing activity of its two primary tick vectors for this region Dermacentor variabilis and Amblyomma americanum $[2,10]$. Likewise, the majority of the sporadic human tularemia cases exhibit a similar spring-summer seasonal pattern; however, approximately $10 \%$ of human tularemia in Oklahoma occurs during the months of November through February when its two primary tick vectors are the least active [11,12]. Many of the cases of human tularemia during this period are attributed to direct transmission from infected rabbits associated with hunting activities [11,12]. However, Hopla noted that some cases of human tularemia during the months when its primary tick vectors are inactive are nevertheless associated with tick transmission [13].

Ixodes scapularis is the third most abundant tick species in the south-central United States. The host preference of adult I. scapularis for large mammals including humans and the coincidence of questing activity with the fall-winter incidence of human tularemia supports its potential as a vector [14]. Domestic cats are highly susceptible to tularemia and the disease incidence in cats shows a bimodal pattern of springsummer and fall maxima [3]. Cats contract the disease by preying on tularemia infected small mammals and the disease during the spring-summer and fall peak incidence is indicative of concurrent tularemia infection cycle in small mammals [3]. Immature stages of I. scapularis seeks small mammals including rabbits and white footed mice as hosts; however, the host seeking activity of the immature $I$. scapularis is predominantly during summer months in the south-central tularemia endemic region [14].

To more fully characterize the potential for I. scapularis to serve as a vector for tularemia, colony-reared I. scapularis 
larvae, nymphs, and adults were infected via artificial capillary tube feeding of tick meal containing $F$. tularensis subspecies holarctica strain LVS to generate sufficient number of infected ticks to allow assessment of quanta and duration of colonization, transstadial transmission, tissue distribution, and secretion in tick saliva.

\section{Materials and Methods}

\section{Ticks, bacterial strain and growth conditions}

I. scapularis larvae, nymphs, and adults were obtained from the Tick Rearing Facility, Department of Entomology and Plant Pathology, Oklahoma State University (Stillwater, OK). Larvae and nymphs were collected after they were fed to repletion on rabbits. Unfed flat adult ticks were used for determination of quanta and tissue distribution of LVS. Adult ticks used for salivary induction experiments were partially fed on sheep for five to six days. Francisella tularensis subsp. holarctica strain LVS was supplied by the Oklahoma State Department of Health. All chemicals used in the study were purchased from Sigma (St Louis, MO) unless indicated otherwise.

\section{Capillary tube feeding of larvae, nymphs and adult ticks}

The ticks were surface disinfected by washing in $30 \%$ hydrogen peroxide, distilled water and $70 \%$ isopropyl alcohol for 5 seconds each. After the washing, adult ticks were immobilized dorsal side up on adhesive part of duct tape placed over doublesided adhesive tape (3M Scotch brand, St Paul, MN) in a 100 by $15 \mathrm{~mm}$ Petri dish base. Ticks were then further immobilized by applying single-sided adhesive tape over $1 / 4^{\text {th }}$ of the caudal region. Larvae and nymphs were immobilized with their dorsal side down on double-sided adhesive tape on dental wax (Electron Microscopy Sciences, Fort Washington, PA). For capillary tube feeding, $10 \mu \mathrm{L}$ (internal diameter of $0.55 \mathrm{~mm}$ ) glass capillary tubes (Drummond Scientific Company, Broomall, PA) were used for larvae and nymphs while $35 \mu \mathrm{L}$ (internal diameter of $0.79 \mathrm{~mm}$ ) glass capillary tubes were used for adult ticks. The tick meal inoculum contained 1-10 $10^{7} \mathrm{CFU} / \mathrm{mL}$ Francisella tularensis subsp. holarctica strain LVS. Detailed description of the preparation of inocula and growth of LVS has been described earlier [15]. After feeding, ticks were either surface disinfected by washing in $30 \%$ hydrogen peroxide, distilled water and $70 \%$ isopropyl alcohol for 5 seconds and minced for determination of CFUs or were maintained in $8 \mathrm{oz}$. paper cup containers with plastic lids and kept in a humidity chamber (relative humidity of $>90 \%$ ) at $22 \pm 1{ }^{\circ} \mathrm{C}$ (unless specified otherwise) with automated artificial lighting to simulate a 12 $\mathrm{h}$ day-night cycle. Determination of CFU per tick was done at one day post-capillary feeding and then at an interval of 7 days. To determine the bacterial numbers in ticks at various times of colonization, individual ticks were minced with a scalpel blade, bacteria extracted by incubation in PBS containing $64 \mu \mathrm{g} / \mathrm{mL}$ ampicillin for $2 \mathrm{~h}$ at room temperature on a rotor platform mixer (Boekel Scientific, Feasterville, PA.), serially diluted in PBS containing $64 \mu \mathrm{g} / \mathrm{mL}$ ampicillin, and plated on chocolate agar plates for CFU determination following incubation at $37{ }^{\circ} \mathrm{C}$ in $5 \% \mathrm{CO}_{2}$ for $72 \mathrm{~h}$. To determine the bacterial numbers in tissues at varying periods of post-capillary tube feeding, individual ticks were dissected into gut and salivary glands under sterile conditions using a dissecting microscope. The tick tissues were placed in PBS containing $64 \mu \mathrm{g} / \mathrm{mL}$ ampicillin and processed as described above for whole ticks. Hemolymph from ticks was collected into sterile glass capillary tubes from the cut ends of the tick's leg. Figure 1 schematically represents the details of the capillary feeding protocol including the number of ticks used and timeline of feeding. On days one through 175 postcapillary feeding, batches of 15 or more ticks were assayed individually for LVS.

\section{Real-time quantitative PCR}

Real- time quantitative PCR was done to determine presence of viable, but non-culturable LVS or LVS DNA in ticks after capillary feeding compared to those which were positive by microbial culture. Real-time PCR setup has been described earlier [16].

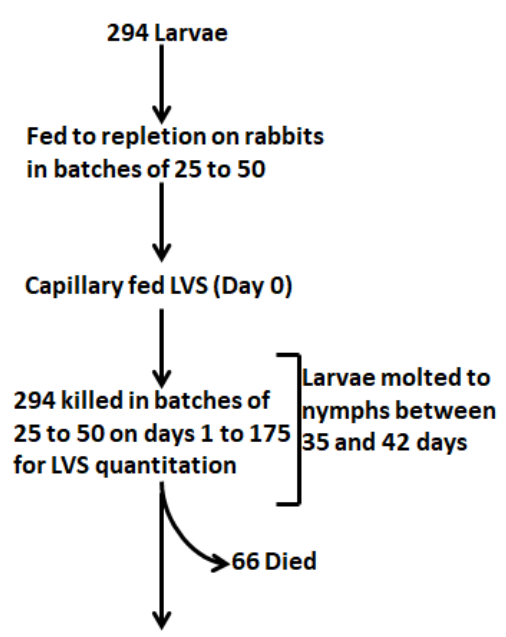

$108(+$ LVS $) / 228$


Figure 1. Quanta and duration of colonization and transstadial transmission of LVS experimental design.

Larvae, nymphs and adults I. scapularis in batches of various sizes were capillary tube fed tick meal containing LVS. The overall rate of ticks positive for LVS for each tick life cycle stage capillary tube fed LVS is given as total number of ticks which were positive for LVS (+LVS)/total number of ticks tested. 


\section{Intra-hemocoelic inoculation and salivary induction in ticks}

To determine the presence of LVS in tick saliva, partially fed adult ticks were inoculated with $1 \mu \mathrm{L}$ of inoculum containing $1 \times 10^{8} \mathrm{CFU} / \mathrm{mL}$ of $F$. tularensis subsp. holarctica strain LVS in PBS injected intra hemocoel in the ventral region of the tick, medial to the caudal most coxa using $10 \mu \mathrm{L}$ custom made Hamilton syringe with a 0.5 inch, 33 gauge needle (Hamilton Company, Reno, NV). For inducing salivary secretion in ticks that were colonized with LVS via capillary tube-feeding, partially fed female ticks were fed with LVS $\left(1 \times 10^{7} \mathrm{CFU} / \mathrm{mL}\right)$ via capillary tube and were held in humidity chamber at $27^{\circ} \mathrm{C}$. Two days post-capillary tube feeding, the ticks were induced to secrete saliva. Partially-fed LVS-colonized adult ticks were immobilized dorsal side up on the sticky part of duct tape placed on a double-sided adhesive tape. Chemical induction and collection of saliva has been described earlier $[15,16]$.

\section{Statistical analysis}

$F$. tularensis colonization in different groups of $I$. scapularis ticks during transstadial transmission from larva to nymphs, and nymph to adult were compared by using 1-way analysis of variance on log-transformed data followed by pairwise multiple comparison of mean CFU value using Holm-Sidak tests. Overall significance level for Holm-Sidak tests was $P=0.05$. All statistical analyses were performed with SigmaPlot v11.0 software package (Systat Software Inc., Chicago, IL).

\section{Results}

\section{LVS colonization of I. scapularis larvae and transstadia transmission to molted nymphs}

At one-day post-capillary tube feeding, $100 \%$ of larvae exhibited colonization as detected by microbial culture with a mean colonization level of $1.9 \pm 1.8 \times 10^{3} \mathrm{CFU} /$ larva (Figure 2; limit of detection was $2 \mathrm{CFU} /$ larva). The colonization level per larvae increased at 7 days post-capillary feeding to $5.3 \pm$ $7.3 \times 10^{4} \mathrm{CFU} /$ larva (unadjusted $P<0.01$ ), suggesting that the bacterium had proliferated following the initial ingestion. For our experimental holding conditions $(\mathrm{RH}=95 \%$ and mean room temperature of $22 \pm 1{ }^{\circ} \mathrm{C}$ ), the larvae molted to nymphs between days 35 to 42 post-capillary tube feeding (Figure 2A). A significant decline in the percent of larvae colonized was observed with the onset of molting at 35 days, declining markedly to 0 to $25 \%$ for sampling days 56 through 175 post capillary feeding. Although only $7 / 51$ (14\%) molted nymphs (Figure 2B) were positive by microbial culture for $F$. tularensis
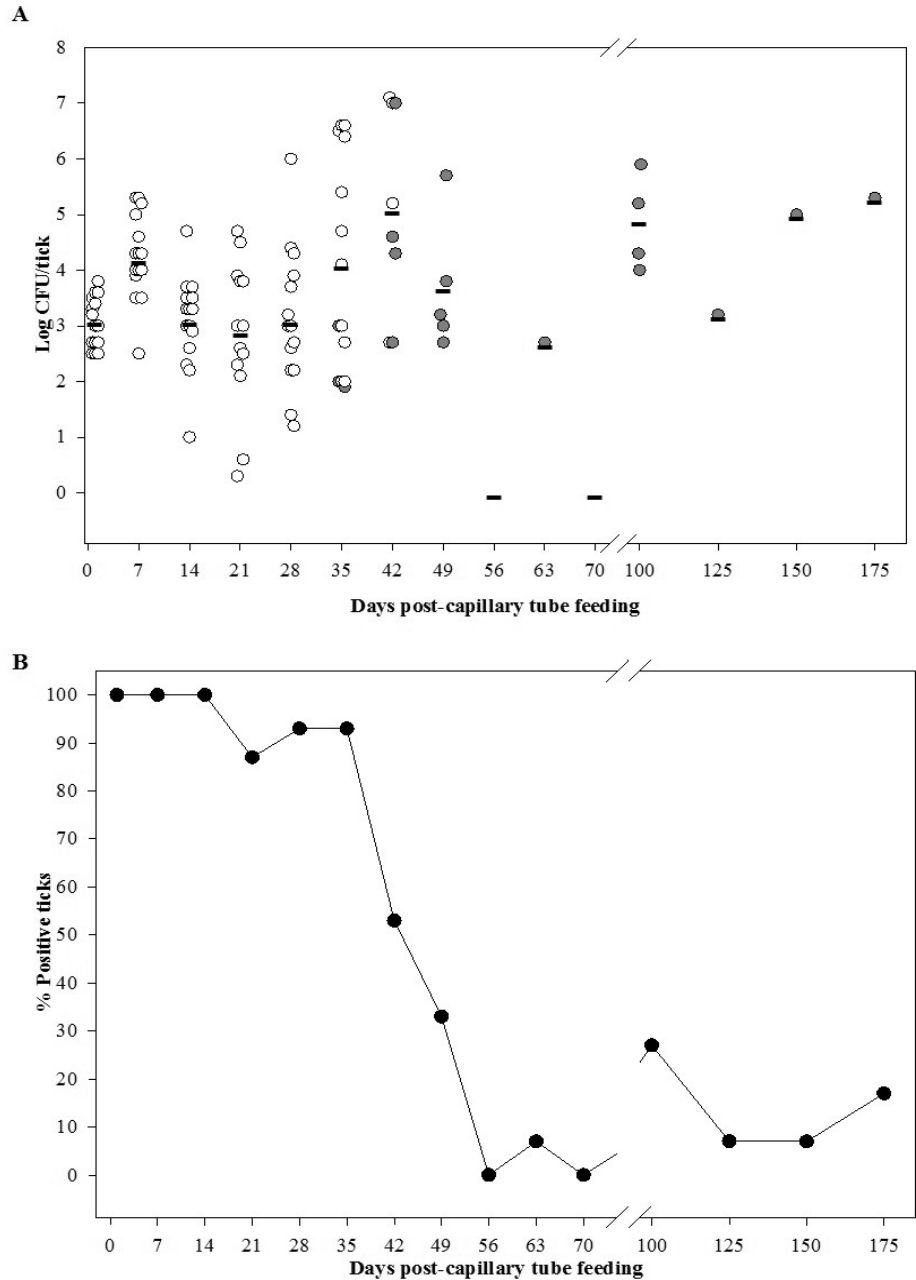

Figure 2. LVS is transmitted transstadially from larvae to nymphs.

A) Open circles are capillary tube fed larvae and filled circles are molted nymphs. The calculated mean CFU/tick for colonized larvae and molted nymphs for each time point is represented by the horizontal line. For each time point up to day 150, $n$ was 15 . For day 175, $n$ was 6 . B) Percentage of colonized ticks in the same experiment. 
from 100 through 175 days post-capillary tube feeding, using RT-PCR, $17 / 51$ (33\%) of these nymphs were positive for $F$. tularensis (Table 1; limit of detection was 5 genome equivalent/ nymph). The difference between LVS quanta determined by culture versus PCR suggests that either LVS may have assumed a viable but not culturable state or that nymphs had cleared the infection but remnant LVS DNA remained in nymphs which was detected by PCR.

Table 1. Comparison of LVS Detection Using Microbial Culture versus RT-PCR.

\begin{tabular}{ccccc}
\hline $\begin{array}{c}\text { Days Post- } \\
\text { Capillary } \\
\text { Feeding }\end{array}$ & \multicolumn{2}{c}{ Molted Nymphs } & \multicolumn{2}{c}{ Molted Adults } \\
\cline { 2 - 5 } & $\begin{array}{c}\text { Microbial } \\
\text { Culture } \\
\text { Positive }\end{array}$ & $\begin{array}{c}\text { RT-PCR } \\
\text { Positive }\end{array}$ & $\begin{array}{c}\text { Microbial Culture } \\
\text { Positive }\end{array}$ & $\begin{array}{c}\text { RT-PCR } \\
\text { Positive }\end{array}$ \\
\hline 100 & $4 / 15$ & $5 / 15$ & $1 / 15$ & $4 / 15$ \\
125 & $1 / 15$ & $2 / 15$ & $0 / 15$ & $0 / 15$ \\
150 & $1 / 15$ & $4 / 15$ & $0 / 15$ & $2 / 15$ \\
175 & $1 / 6$ & $6 / 6$ & $N^{a}$ & $N^{a}$ \\
Total & $7 / 51(14 \%)$ & $17 / 51(33 \%)$ & $1 / 45(2 \%)$ & $6 / 45(13 \%)$ \\
\hline
\end{tabular}

a Data not collected.

\section{LVS colonization of I. scapularis nymphs and transstadial transmission to molted adults}

At one-day post-capillary tube feeding, $100 \%$ of nymphs were colonized with a mean level of $1.1 \pm 1.2 \times 10^{4} \mathrm{CFU} /$ nymph (Figure 3A). The bacterial count per tick maintained at the same level until day 7 post-capillary tube feeding and then decline until molting to adults at around 42 days post-capillary tube feeding. Although $F$. tularensis was transstadially transmitted to adult ticks, only $1 / 45(2 \%)$ of the molted ticks were positive for the bacterium after 100 day post-capillary tube feeding by microbial culture (Figure 3B); however, a slightly higher percentage $6 / 45(13 \%)$ of adults molted from nymphs were positive for $F$. tularensis by RT-PCR (Table 1).

\section{Tissue localization of LVS in I. scapularis adults}

To determine the dissemination of $F$. tularensis in adult tick tissues following colonization by ingestion, unfed flat adult ticks were capillary tube fed with tick meal and subsequently dissected on various days post-capillary feeding to determine

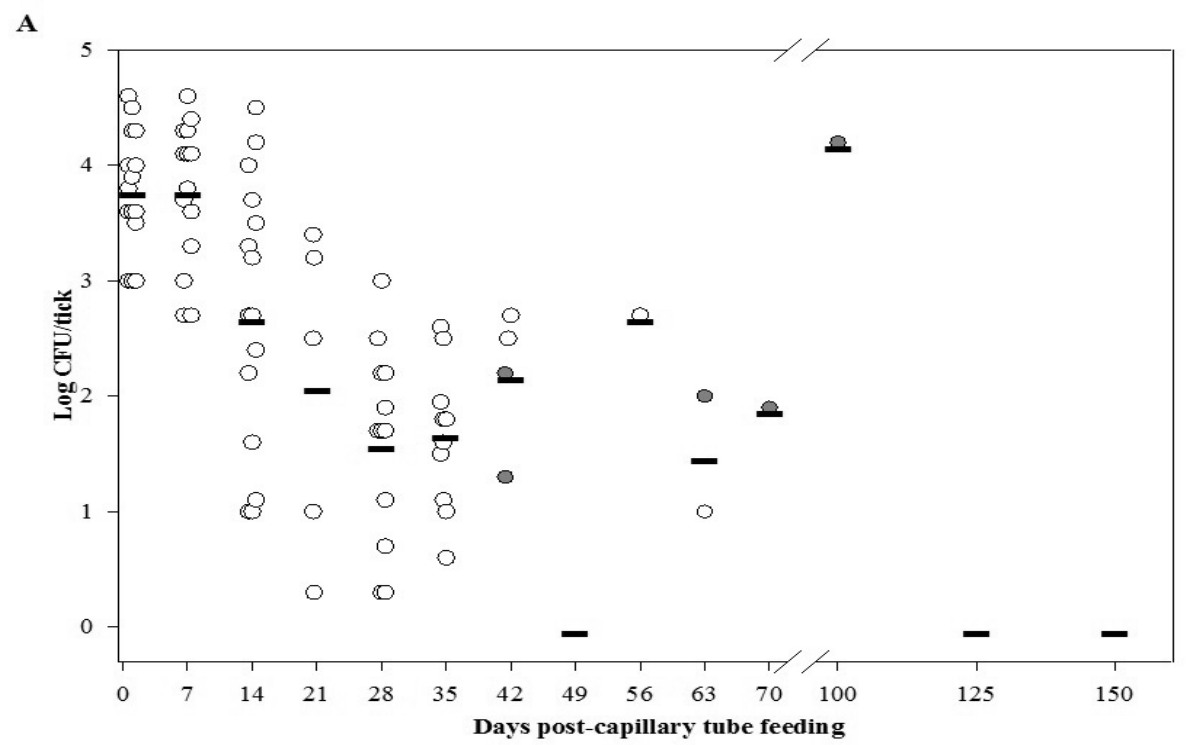

B

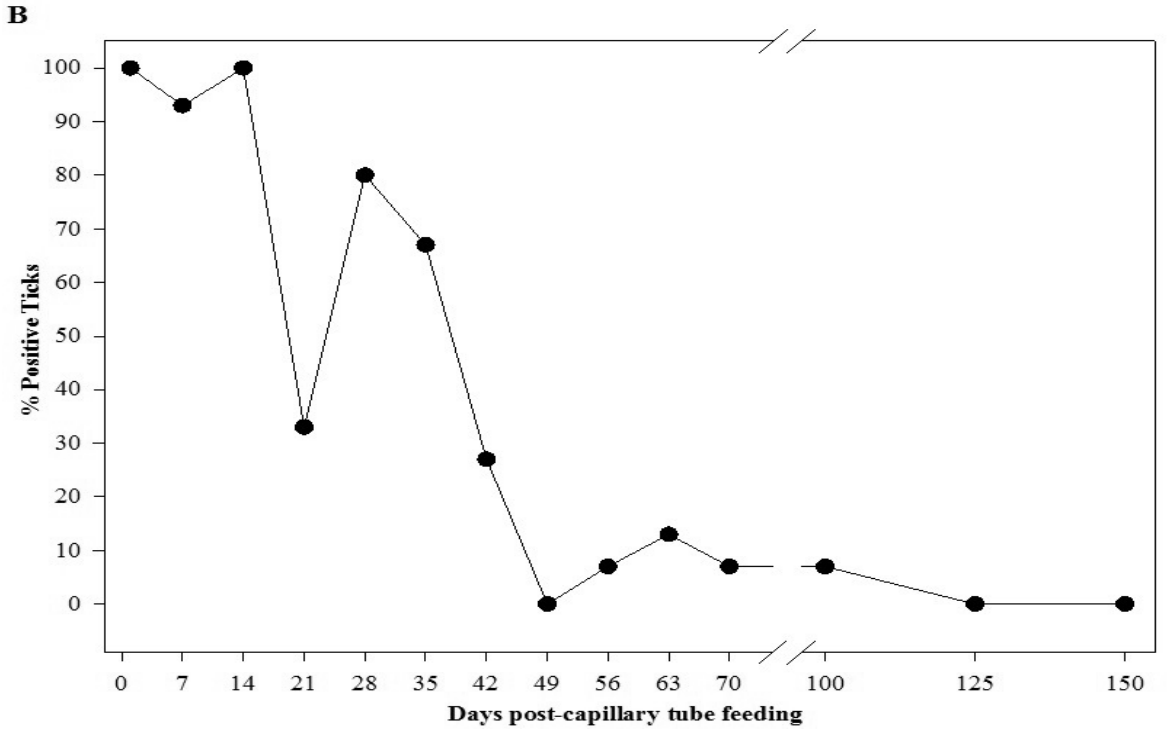

Figure 3. LVS is transmitted transstadially from nymphs to adults.

A) Open circles are capillary tube fed nymphs and filled circles are molted adults. The calculated mean CFU/tick for colonized nymphs and molted adults for each time point is represented by the horizontal line. For each time point up to day 150, $n$ was 15. B) Percentage of colonized ticks in the same experiment. 
the tissue dissemination of LVS. In adult I. scapularis, $100 \%$ $(n=27)$ remained positive by microbial culture for LVS from day one through day 28 post-capillary tube feeding (Figure 4). By day 7 post-capillary tube feeding, LVS was detected in hemolymph in $60 \%(3 / 5)$ of ticks sampled, with LVS detected in hemolymph of at least $40 \%(2 / 5)$ of ticks through day 28 . LVS was not detected in salivary gland until day 14 post-capillary tube feeding, when $33 \%$ (2/6) of ticks sampled had LVS in their salivary glands, and LVS was detected in at least $40 \%(2 / 5)$ of tick salivary glands through day 28 .

\section{LVS secretion in I. scapularis saliva}

To determine whether LVS disseminated to salivary glands of adult ticks is secreted in saliva, partially fed adult female $I$. scapularis were inoculated i.h. with LVS and secretion in saliva determined. Post i.h. inoculation, LVS disseminated from the hemolymph into gut and salivary gland and was secreted in the saliva in $67 \%$ of the ticks $(2 / 3)$ within two days post-injection with a mean level of $1.8 \pm 2.8 \times 10^{3} \mathrm{CFU} / \mu \mathrm{L}$ of saliva.

\section{Discussion}

In the south-central U.S. tularemia endemic region, the springsummer enzootic tularemia cycle is maintained by $D$. variabilis and $A$. americanum tick-vectored infections of small mammals, particularly rabbits. Both ticks species are infected with $F$. tularensis in nature, can maintain the bacterium from immature stages to the adult, act as experimental vectors for $F$. tularensis transmission, and have the potential to serve as inter-epizootic reservoir of $F$. tularensis [2,15-17]. Based on the seasonal questing activity and feeding habits of these tick species, it is likely that adult $D$. variabilis and nymphal $A$. americanum are the two stages important as a vector for transmitting $F$. tularensis to humans $[2,15,16]$. While D. variabilis and A. americanum are important vectors for the majority of human tularemia which occur during the spring-summer months, sporadic human tularemia cases are also reported during the fall-winter months from November to February in this endemic region $[11,12]$. Most of these fall-winter human tularemia cases result from direct contact with infected rabbits associated with hunting; however, some cases result from tick transmission [11]. Hopla proposed that I. scapularis was the tick species responsible for these tick associated fall-winter human tularemia cases [13]. He found that larval I. scapularis could acquire F. tularensis, maintain infection through the adult stage, and transmit the infection experimentally to laboratory animals. Hopla also observed that wild caught nymphal I. scapularis were naturally infected with $F$. tularensis and could transmit the infection experimentally [13].

The research reported herein extends that of Hopla's by more fully characterizing the duration and quanta of infection in $I$. scapularis larvae and nymphs and the tissue distribution and secretion in saliva of $F$. tularensis in adult $I$. scapularis. Similar to what we have previously reported for $F$. tularensis infection of $D$. variabilis and $A$. americanum using the same experimental approach, I. scapularis exhibited an increase in mean CFU of $F$. tularensis /tick after molting compared to just prior to molting $[15,16]$. In contrast to what we observed in $D$. variabilis and $A$. americanum in which transstadial transmission was observed for $>50 \%$ molted progeny, transstadial transmission was $<50 \%$ in I. scapularis nymphs and adults; however, for all of the ixodid ticks species and stages tested, infection was maintained in some percentage of infected ticks through the longest time examined from 42 to 175 day post-capillary tube feeding. Tissue dissemination of $F$. tularensis infection from the gut to hemocoel and other tissues occurred in $<7$ days in $A$. americanum adults, between 7 and 14 days in I. scapularis, and between 14 to 21 days in D. variabilis. Extension of infection from the hemocoel to salivary glands and secretion in saliva occurred within 2 days in all three ixodid ticks, demonstrating that the dissemination from gut to hemocoel may be a rate-limiting step for vector competency for transmission of tularemia by ixodid ticks.

Although the experimental work reported by Hopla and herein demonstrate the capacity of I. scapularis to act as a vector for transmission of $F$. tularensis, the question remains as to whether I. scapularis actually functions as a vector for the enzootic tularemia cycle or as a vector for sporadic human tularemia? The peak questing period for I. scapularis larvae and nymphs is spring-summer coincident with the peak of enzootic tularemia. Although the feeding preference for I. scapularis larvae and
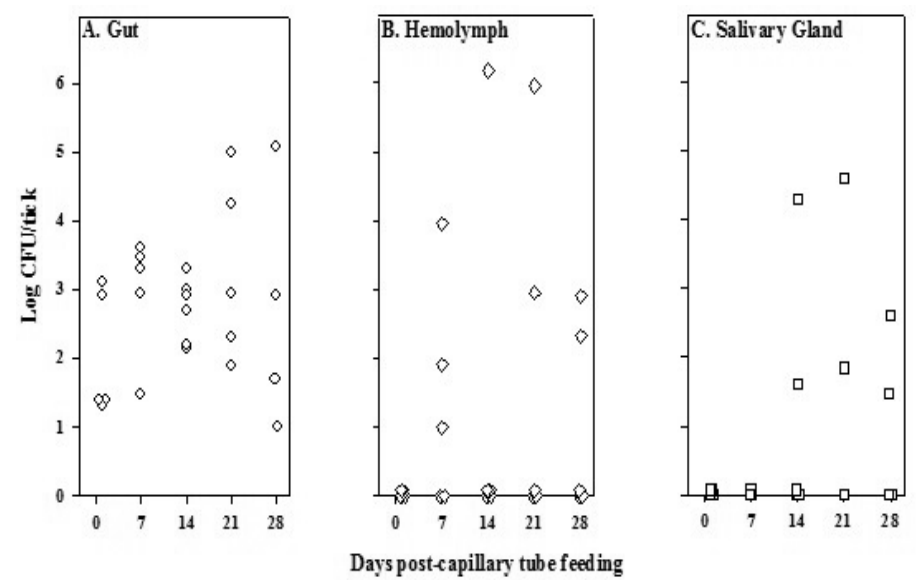

Figure 4. Tissue dissemination of LVS in adult I. scapularis.

A) Open circles are LVS in gut. B) Open diamonds are LVS in hemolymph. C) Open squares are LVS in salivary gland. Total $n$ was 27 with $n$ for each time point and tissue ranging from 3 to 6 . 
nymphs in the south-central U.S. tularemia endemic region is for reptiles and birds, the immature ticks at a lower prevalence can feed on small mammals thought to be the amplifying hosts for maintaining the enzootic tularemia cycle [14,17]. In contrast to the spring-summer questing period and bird-reptile feeding preference for I. scapularis larvae and nymphs, adult I. scapularis exhibit a fall-winter questing period with feeding preference for large mammal such as canids and deer [14].

\section{Conclusion}

Using the capillary feeding method of infecting ticks, we showed that $F$. tularensis strain LVS multiply and maintain parasitism in I. scapularis. Although the number of long-term infected ticks were much lower than what was observed with respect to $D$. variabilis and $A$. americanum, a small percentage of ticks still maintained a high quanta of the bacteria sufficient for transmission to susceptible hosts. From our studies, we confirm the potential for I. scapularis adults to serve as bridging vector for transmission of sporadic human tularemia during the fall-winter months in the south-central United States tularemia endemic region and I. scapularis larvae and nymphs to serve as a vector for small mammals in the summer enzootic tularemia cycle; however, based on the feeding preference of these tick species I. scapularis may not play a prominent role in the ecology of tularemia.

\section{References}

1. Keim PS, Wagner DM. Humans and evolutionary and ecological forces shaped the phylogeography of recently emerged diseases. Nat Rev Microbiol. 2009;7(11):813-21.

2. Eisen LA. Call for renewed research on tick-borne Francisella tularensis in the Arkansas-Missouri primary national focus of tularemia in humans. J Med Entomol. 2007;44(3):389-97.

3. Mani RJ, Morton RJ, Clinkenbeard KD. Ecology of tularemia in central US endemic region. Curr Trop Med Rep. 2016;3:75-79.

4. Kugeler KJ, Mead PS, Janusz AM, et al. Molecular Epidemiology of Francisella tularensis in the United States. Clin Infect Dis. 2009;48(7):863-70.

5. Staples JE, Kubota KA, Chalcraft LG, et al. Epidemiologic and molecular analysis of human tularemia, United States, 1964-2004. Emerg Infect Dis. 2006;12(7):1113-8.

6. Petersen JM, Molins CR. Subpopulations of Francisella tularensis ssp. tularensis and holarctica: identification and associated epidemiology. Future Microbiol. 2010;5:649-61.

7. Snowden J, Stovall S. Tularemia: retrospective review of 10 years' experience in Arkansas. Clin Pediatr (Phila). 2011;50(1):64-8.

8. Sjostedt A. Tularemia: history, epidemiology, pathogen physiology, and clinical manifestations. Ann N Y Acad Sci. 2007;1105:1-29.

9. Ellis J, Oyston PC, Green M, et al. Tularemia. Clin Microbiol Rev. 2002;15(4):631-46.

10. Jellison WL. Tularemia in North America. 1974, Missoula, Montana: University of Montana.

11. Assal NR, Lindeman RD, Carpenter RL. Epidemiologic study on reported human tularemia in Oklahoma, 1944-65. J Okla State Med Assoc. 1968;61(3):120-4.

12. Rohrbach BW, Westerman E, Istre GR. Epidemiology and clinical characteristics of tularemia in Oklahoma, 1979 to 1985. South Med J. 1991;84(9):1091-6.

13. Hopla CE. Ixodes scapularis as a vector of tularemia organisms in the southern United States. Proceedings of the 11th International Congress of Entomology. 1962 Vienna, Austria.

14. Kollars TM, Oliver JH, Kollars PG, et al. Seasonal activity and host associations of Ixodes scapularis (Acari: Ixodidae) in southeastern Missouri. J Med Entomol. 1999;36(6):720-6.

15. Mani RJ, Reichard MV, Morton RJ, et al. Biology of Francisella tularensis subspecies holarctica live vaccine strain in the tick vector Dermacentor variabilis. PLoS One. 2012;7(4):e35441.

16. Mani RJ, Metcalf JA, Clinkenbeard KD. Amblyomma americanum as a bridging vector for human infection with Francisella tularensis. PLoS One. 2015;10(6):e0130513.

17. Hopla CE. The ecology of tularemia. Adv Vet Sci Comp Med. 1974;18(0): 25-53.

\section{*Correspondence to:}

Rinosh J. Mani

Section Chief (Bacteriology/Mycology), Academic Specialist

Veterinary Diagnostic Laboratory

College of Veterinary Medicine

Michigan State University

Lansing, MI, USA

Tel: (517) 353-9053

E-mail:manirino@msu.edu 